\title{
Fuzzy Multi-object Optimization Design of Helical Gear Drive
} Xiao-xing Liu ${ }^{1}$, Qi-ying Pan ${ }^{2 *}$

${ }^{1}$ College of Mechanical and Electrical Engineering, Kunming University of Science and Technology, Kunming,Yunnan, 650093, China

${ }^{2}$ College of Mechanical and Electrical Engineering, Kunming University of Science and Technology, Kunming,Yunnan, 650093, China

Keywords: Helical Gear Drive, Reducer, Multi-objective Optimization Design, Fuzzy Mathematics.

\begin{abstract}
Fuzzy factors in the helical gear reducer design are analyzed, and a multi-objective optimization model with minimum volume and maximum bearing capacity is established in this paper. For multi-objectives, single-objective optimal solutions are fuzzified to form fuzzy sets to establish the membership functions of the fuzzy sets by taking the fuzziness between the single objectives and between the single objectives and multiple objectives into consideration, and multi-objective optimal solutions are obtained by maximizing the membership functions of the fuzzy set intersection. For an actual helical gear reducer, the fuzzy theory and method are applied to obtain results, and the validness of the method is demonstrated by comparing such results with the conventional design data.
\end{abstract}

\section{Introduction}

Volume, weight and bearing capacity are the important indexes for evaluating the performance of helical gear reducer and these indexes depends on the design and selection of the transmission parameters such as module $\mathrm{m}$, width of gear $\mathrm{b}$, teeth number of gear $\mathrm{zl}$, helix angleßetc. The optimization design solutions targeted at minimum volume in the priorart[1] cannot reflect the actual operation conditions, as they do not take the fuzzy factors in the design into consideration and have single objective, and by adopting such solutions, the real optimization solution is highly possible to be missed to obtain the satisfactory comprehensive effects. In this paper, the multi-objective fuzzy optimization model with minimum volume and maximum bearing capacity is established by combining with the optimization techniques and adopting the theory and method of fuzzy mathematics. Optimal level cut-set method is introduced to conduct comprehensive evaluation on constraining fuzziness and convert the fuzzy constraint into general constraint according to the actual conditions and requirements; for multi-objectives, single-objective optimal solutions are fuzzified to form fuzzy sets to establish the membership functions of the fuzzy sets by taking the fuzziness between the single objectives and between the single objectives and multiple objectives into consideration, and multi-objective optimal solutions are obtained by maximizing the membership functions of the fuzzy set intersection.

\section{Establishing Multi-objective Fuzzy Optimization}

\section{Objective Functions and Design Variables}

The objective of the optimization is the confining dual-objective model with minimum volume and maximum bearing capacity under the condition of ensuring transmission ratio and various confining conditions, and the minimum volume is taken as the optimization objective, at the premise of meeting the application requirements, which mean[2]s:

$$
\mathrm{F} 1(\mathrm{x})=0.785 \mathrm{~m} 2 \mathrm{bz} 12(1+\mathrm{i} 2) / \cos 2 \beta
$$

Where: $\mathrm{m}$-modules of gear

$\mathrm{b}$ - width of gear

$\mathrm{i}$ - transmission ratio 
$\mathrm{z} 1$-teeth number of gear

$\beta$--helix angle

For bearing capacity, the input torque $\mathrm{T} 1 \mathrm{can}$ be taken as objective function:

$$
\mathrm{F} 2(\mathrm{x})=\mathrm{T} 1
$$

Obviously, F1(x) and F2(x) are both subjected to the confining effects of the parameters $\mathrm{m}, \mathrm{b}, \mathrm{z} 1$ and $\mathrm{T} 1$, thus, the design variable can be taken as:

$$
\mathrm{X}=\left[\begin{array}{lllll}
\mathrm{m} & \mathrm{b} & \mathrm{z} 1 & \mathrm{~T} 1 & \beta
\end{array}\right] \mathrm{T}=\left[\begin{array}{lllll}
\mathrm{x} 1 & \mathrm{x} 2 & \mathrm{x} 3 & \mathrm{x} 4 & \mathrm{x} 5
\end{array}\right] \mathrm{T}
$$

So, the objective function can be established as:

$$
\mathrm{F} 1(\mathrm{x})=0.785 \times 12 \times 2 \times 32(1+\mathrm{i} 2) / \cos 2 \times 5
$$

$$
F_{2}(x)=x_{4}
$$

\section{Confining Conditions}

From the perspectives of actual conditions of the gear drive, the confining conditions can be divided into stress constraints and boundary constraints. Stress constraints include constraint of contact strength of gear tooth face and constraint of bending strength of tooth root, and the boundary constraints include the constraints on value taking for modules, width of gear and teeth number of gear. For these constraints, the determination of the stress constraints has significant fuzziness and they shall be deemed as fuzzy constraints, while other constraints can be taken as general constraints.

\section{Stress Constraints}

The constraint of contact strength of gear tooth face and constraint of bending strength of tooth root are respectively shown as follow[3]s:

$$
\begin{gathered}
g_{1}(x)=Z_{E} \cdot Z_{H} Z \text { в } \sqrt{\frac{2 k T_{1}(u+1)}{b d 1^{2} u}}-\left[\begin{array}{c}
\sigma \\
\sim
\end{array}\right]_{H} \leq 0 \\
g_{2}(x)=\frac{2 k T 1}{d_{1} m b} Y_{F a} Y_{s a}-\left[\begin{array}{c}
\sigma \\
\sim
\end{array}\right]_{F} \leq 0
\end{gathered}
$$

Where: ZE - elastic factor of material,

$\mathrm{ZH}$ - region factor,

ZB-helix angle factor,

$\mathrm{k}$ - load coefficient,

$\mathrm{T} 1$ - torque of worm gear, $\mathrm{N} \cdot \mathrm{mm}$

$\left[\begin{array}{c}\sigma \\ \sim\end{array}\right]_{H}$

$[\sim]_{H}$ - allowable contact stress with fuzziness

$\mathrm{YFa}$ - tooth form coefficient of gear

Ysa-correct coefficient of gear

$\left[\begin{array}{c}\sigma \\ \sim\end{array}\right]$

- allowable bending stress with fuzziness

\section{Boundary Constraints}

For module limit $(1.5 \leq \mathrm{m} \leq 4)$ 
For limit of width of gear $(30 \leq b \leq 80)$

For limit of number of teeth of gear $(18 \leq \mathrm{z} 1 \leq 28)$

For torque limit $(\mathrm{Tmin} \leq \mathrm{T} 1 \leq \mathrm{Tmax})$

Helix angle limit $(100 \leq \beta \leq 200)$

Thus, the multi-objective optimization module of reducer is obtained by:

Solving $X=\left[\begin{array}{lllll}\mathrm{m} & \mathrm{b} & \mathrm{z} 1 & \mathrm{~T} 1 & \beta\end{array}\right] \mathrm{T}=\left[\begin{array}{lllll}\mathrm{x} 1 & \mathrm{x} 2 & \mathrm{x} 3 & \mathrm{x} 4 & \mathrm{x} 5\end{array}\right] \mathrm{T}$ to make $\operatorname{minF} 1(\mathrm{x})$ and $\operatorname{maxF}(\mathrm{x})$ meet the following requirement:

$$
\text { S.T. } g u(x) \leq 0(\mathrm{u}=1,2, \square \quad \text { and } 7)
$$

\section{Dealing with Fuzzy Constraints ${ }^{[4]}$}

For the confining conditions g1(x) and g2(x), the values of allowable stresses $\left[\begin{array}{c}\sigma \\ \sim\end{array}\right]_{H}$ and $\left[\begin{array}{c}\sigma \\ \sim\end{array}\right]_{F}$ are taken within certain range, and the determination of the specific values depends on various fuzzy factors. By assuming the upper and lower limits of $\left[\begin{array}{c}\sigma \\ \sim\end{array}\right]_{H}$ and $\left[\begin{array}{c}\sigma \\ \sim\end{array}\right]_{F}$ as $\left[\begin{array}{c}- \\ \sigma\end{array}\right]_{H},\left[\begin{array}{c}\sigma \\ -\end{array}\right]_{H}$, $\left[\begin{array}{c}- \\ \sigma\end{array}\right]_{F}$ and $\left[\begin{array}{c}\sigma \\ -\end{array}\right]_{F}$, level cut-set is introduced, and the corresponding allowable stresses are respectively as follows:

$$
\begin{aligned}
& {\left[\begin{array}{l}
\sigma \\
\sim
\end{array}\right]_{H}=\left[\begin{array}{c}
- \\
\sigma
\end{array}\right]_{H}-\left(\left[\begin{array}{c}
\sigma \\
\sigma
\end{array}\right]_{H}-\left[\begin{array}{c}
\sigma \\
-
\end{array}\right]_{H}\right) V} \\
& {\left[\begin{array}{l}
\sigma \\
\sim
\end{array}\right]_{F}=\left[\begin{array}{l}
- \\
\sigma
\end{array}\right]_{F}-\left(\left[\begin{array}{l}
- \\
\sigma
\end{array}\right]_{F}-\left[\begin{array}{l}
\sigma \\
-
\end{array}\right]_{F}\right) V}
\end{aligned}
$$

$V \in[0,1]$

\section{Solution of Optimization Module}

The solution steps of multi-objective optimization model by adopting fuzzy theory and method as determined by Formula (16) are as follows:

1) The optimal values $f_{1}^{*}$ and $f_{2}^{*}$ as well as the worst values $\bar{f}$ and $\bar{f}_{2}$ of the objectives are obtained by general single objective optimization method, and obviously, the following can be obtained:

$$
\begin{aligned}
& f_{1}^{*} \leq f_{1}(x) \leq \overline{f_{1}} \\
& \bar{f}_{2} \leq f_{2}(x) \leq f_{2}{ }^{*}
\end{aligned}
$$

2) The optimal solutions are fuzzified to establish the membership functions of the optimal solutions:

$$
\tilde{N}_{1}(x)=\left[\frac{\bar{f}_{1}-f_{1}(x)}{\bar{f}_{1}-f_{1}^{*}}\right]^{q}
$$




$$
\tilde{N}_{2}(x)=\left[\frac{f_{2}(x)-\bar{f}_{2}}{f_{2}^{*}-\bar{f}_{2}}\right]^{q}
$$

Where: $\tilde{N}_{1}(x)$ and $\tilde{N}_{2}(x) \in[0,1]$

3) Instrumental variable $\mathrm{a}$ is introduced to convert the multi-objective issue into the single-objective issue, which means that:

$\mathrm{X}==\left[\begin{array}{llllll}\mathrm{x} 1 & \mathrm{x} 2 & \mathrm{x} 3 & \mathrm{x} 4 & \mathrm{x} 5 & \mathrm{a}\end{array}\right] \mathrm{T}$ is solved to make max a meet the following:

S.T. $g u(x) \leq 0(u=1,2, \ldots, 7)$

$$
\mathrm{a} \leq \tilde{N}_{1}(x) \text { and } \tilde{N}_{2}(x)
$$

\section{Calculation Example Analysis}

For a motor-driven helical gear reducer, with motor power $\mathrm{P}=40 \mathrm{kw}$, speed $\mathrm{n}=1470 \mathrm{rpm}$, transmission ratio $\mathrm{i}=3.3$ and middling shock load, for which two-way drive is adopted, the little gear is made from $20 \mathrm{CrMnTi}$, the large gear is made from $20 \mathrm{Cr}$, $56 \sim 62 \mathrm{HRC}$ is adopted, the value taking range of $\left[\begin{array}{c}\sigma \\ \sim\end{array}\right]_{H}$ is $1450 \sim 1540 \mathrm{MPa}$, that of $\left[\begin{array}{c}\sigma \\ \sim\end{array}\right]_{F}$ is $448 \sim 504 \mathrm{MPa}$, that of $\mathrm{T} 1$ is 250000 280000N.mm, and the other fuzzy conditions are high design and manufacture levels, good application conditions, large damage and loss, high-quality materials, and high significance of reducer. The cylindrical worm reducer is designed herein based on the minimum volume and maximum bearing capacity.

The optimal level cut-set $V^{*}$ is first determined based on the above-mentioned method to convert the fuzzy constraint into general constraint:

The factor set is $U=$ (high design and manufacture levels, good application conditions, large damage and loss, high-quality materials, and high significance of reducer)

The alternative set is $\mathrm{V}=(0.0,0.1,0.2, \ldots, 0.9$ and 1.0$)$.

The factor weight set is:

$\mathrm{A}=(0.25,0.23,0.20,0.20,0.10$ and 0.02$)$

The optimal level cut-set can be obtained as $V^{*}=0.54$ based on the weighted average method, and is introduced into the formulas (14), (15), (6) and (7) to convert the fuzzy constraint into general constraint.

The best point and worst point obtained from optimization of single objective are:

$$
\begin{array}{lr}
f_{1}^{*}=1196167 \mathrm{~mm} 3 & \bar{f}=9293107 \mathrm{~mm} 3 \\
f_{2}^{*}=280000 \mathrm{Nmm} & \bar{f}_{2}=250000 \mathrm{Nmm}
\end{array}
$$

The established membership functions are as follows (for $\mathrm{q}=1$ ):

$$
\begin{aligned}
& \tilde{N}_{1}(x)=\left[\frac{9293107-f_{1}(x)}{9293107-1196167}\right] \\
& \tilde{N}_{2}(x)=\left[\frac{f_{2}(x)-250000}{280000-250000}\right]
\end{aligned}
$$

The above said results are introduced into the formula (20), and the optimal solutions are $(\mathrm{a}>0.98)$.

$\mathrm{X}=\left[\begin{array}{lllll}2.5 & 60 & 18 & 280000 & 15044^{\prime} 24\end{array}{ }^{\prime \prime}\right], \mathrm{F} 1=1304910 \mathrm{~mm} 3$ and $\mathrm{F} 2=280000 \mathrm{Nmm}$. The volume is reduced by $25.2 \%$ and the bearing capacity is improved by $7.1 \%$ when compared with the original design parameters [3] [3 5019260000 18053'16"](F1 = $1744713 \mathrm{~mm} 3)$.

The comprehensive optimization effect is very remarkable. 


\section{Conclusions}

1) Fuzzy comprehensive evaluation and fuzzy membership function methods are applied in the paper respectively to solve the confining conditions and multi-ob jective fuzziness issues in the cylindrical worm reducer optimization design to make the design results more consistent with the actual conditions, with good comprehensive effect.

2) The results of fuzzy optimization are closely related to the determination of the factor weight set. Grade I fuzzy comprehensive evaluation method is adopted in the paper, though Grade II or even Grade III fuzzy comprehensive evaluation method should be adopted in determination of factor weight set to reduce or eliminate the anthropogenic factors such as experience and point of view which are closely related, as the work amount of calculation will be significantly increased.

3) The calculation example of the paper gives the specific fuzzy conditions. Changes to these conditions will lead to different optimization results (ordinary optimization design method cannot keep the optimization results unchanged in case of changes to fuzzy conditions). Therefore, this fuzzy optimization design is more suitable for the design with many factors and more specific conditions.

\section{References}

[1] Chen Lizhou, et al.,Mechanical Optimization Design, Shanghai Science \& Technology Press, Shanghai, 2012

[2] Sun Heng and Fu Zeshao, Theory of Machines and Mechanism (the fourth edition), Higher Education Press, Beijing, 2008

[3] Yang Kezhen, el at., Foundation of Mechanical Design (the fifth edition), Higher Education Press, Beijing, 2006

[4] Wang Caihua and Song Liantian,Methodology in Fuzzy Mathematics, China Architecture \& Building Press, Beijing, 1988 